\title{
Algunas reflexiones sobre la regulación del financiamiento participativo o crowdfunding
}

\author{
Luis Ernesto Marín Villarán* \\ Juan Diego Cruz Kamiche*
}

\begin{abstract}
Resumen. - Ante el acelerado avance de las innovaciones en el terreno de las fintech, se abre el debate sobre la regulación que debe girar en torno de ellas. En el presente artículo, los autores reflexionan acerca del financiamiento participativo o crowdfunding que actualmente ya es materia de iniciativas tanto en el Congreso de la República como en el Poder Ejecutivo.
\end{abstract}

\begin{abstract}
Given the accelerated progress of innovations in the field of fintech, the debate about the regulation that must revolve around them opens. In this article, the authors reflect on participatory financing or crowdfunding that is currently subject of initiatives both in this administration and Congress.
\end{abstract}




\section{Introducción}

Cada vez es más frecuente escuchar hablar de las "fintech", esto es, empresas que utilizan en forma activa la tecnología para mejorar las actividades financieras. Las "fintech" constituyen toda una revolución en la industria financiera, ya que están cambiando, con mucha innovación y a pasos acelerados, la forma cómo tradicionalmente se ha venido prestando servicios financieros, así como la forma cómo las personas interactuamos a su vez con los proveedores de servicios financieros.

Como suele suceder, la realidad avanza a pasos agigantados, sobretodo en esta nueva industria "fintech", y hoy por hoy existe mucho debate sobre si distintos servicios que son brindados por o a través de las "fintech" deben ser regulados. Siendo la innovación una característica de las "fintech", es entendible el temor a una regulación que no entienda las especiales características y necesidades de las "fintech" y termine obviando los beneficios que este fenómeno puede traer a la industria de servicios financieros, desde un aumento en la inclusión financiera hasta la forma cómo interactuamos diariamente con nuestro banco. No se trata de regular por regular, sino de regular lo que deba ser regulado y, aun así, que una regulación a las "fintech" no termine por matar lo que constituye su principal característica: la innovación.

Si bien podemos dedicar varias páginas a hablar de la regulación de las distintas actividades que son desarrolladas por las "fintech", desde la posibilidad de poder realizar operaciones de compra y venta de moneda desde una app en tiempo real, la utilización del dinero electrónico como mecanismo para la inclusión financiera o plataformas de pago, hasta open banking y la necesidad de que las empresas financieras de hoy "abran" sus APIs (Application Programming Interface) a terceros, en el presente artículo queremos plantear algunas reflexiones respecto a lo que parece será una realidad en el corto plazo: la regulación del financiamiento participativo o crowdfunding, y que ha sido ya materia de algún proyecto presentado al Congreso de la República, mientras que en el Poder Ejecutivo se vienen discutiendo a su vez otros proyectos con la participación del Ministerio de Economía y Finanzas, la Superintendencia del Mercado de Valores y la Superintendencia de Banca, Seguros y Administradoras Privadas de Fondos de Pensiones.

\section{El funcionamiento del Crowdfunding}

La presente sección tiene como finalidad efectuar un análisis apoyado en un enfoque teórico de la estructura del crowdfunding por lo que resulta importante mencionar que en su mayoría la información obtenida en los siguientes numerales guarda relación con su desempeño y experiencias en jurisdicciones extranjeras considerando que actualmente el crowdfunding no es una actividad autorizada por el ordenamiento jurídico peruano. 


\section{i. Marco conceptual}

En nuestro país constantemente se aprueban normas cuya finalidad primordial es promover en su mayoría el acceso de pequeñas y medianas empresas de distintos sectores a diferentes alternativas y/o estructuras de financiamiento y de esa forma contribuir al crecimiento y desarrollo sostenido de la economía nacional ${ }^{1}$. Sin embargo, existen también personas naturales y jurídicas que regularmente requieren financiamiento para desarrollar emprendimientos o materializar ideas que consideran rentables por lo que recurren en algunos casos a herramientas tecnológicas que se encuentren a su alcance, como es el caso de las páginas web, para poder hacer de conocimiento público ello.

En ese orden de ideas nace el crowdfunding como un mecanismo de financiamiento alternativo al sistema bancario y el mercado de valores a través del cual se obtienen los recursos necesarios a fin de desarrollar proyectos, negocios o actividades determinadas o determinables. Para efectos de la identificación del uso de los recursos captados en el marco del mencionado mecanismo de financiamiento se consideran como actividades determinadas aquellas que al momento del ofrecimiento de participación y posterior entrega de los fondos han sido previamente analizadas cada una de las etapas que involucran. Por otro lado, respecto de las actividades determinables se consideran aquellas que se encuentran en una etapa preliminar de planteamiento de propuesta de desarrollo sin haber aun delimitado las etapas o pasos a seguir para su materialización.

Sobre el particular, las Naciones Unidas ha señalado en su publicación del 12 de junio del 2017 denominada "Financing Solutions for Sustainable Development" que en sus inicios el crowdfunding era considerado como un experimento social, sin embargo actualmente es una industria multibillonaria teniendo un ascenso en cifras según señala el organismo multilateral bajo mención que va desde US\$ 1 billón en 2011 a US\$ 34 billones en el 2015. Del mismo modo, se señala en el documento materia de comentario que inicialmente el crowdfunding se encontraba destinado a obtener financiamiento para actividades de caridad, sin embargo actualmente ha ampliado su alcance también a financiamiento para actividades empresariales.

Con referencia al incremento de cifras generadas en el marco del desarrollo del crowdfunding resulta importante mencionar que ello va de la mano con la continua innovación tecnológica en la que día a día estamos inmersos. No es lo mismo

1 Uno de los ejemplos más claros es el mercado alternativo de valores que es un segmento del mercado de valores creado por la Superintendencia del Mercado de Valores - SMV cuyo objetivo es que las pequeñas y medianas empresas ingresen al mercado de valores teniendo un régimen sancionador especial, régimen de tarifas y contribuciones menores, reducción de costos y tiempo en el trámite de inscripción de los valores objeto de oferta en el Registro Público del Mercado de Valores y Registro de la Bolsa de Valores de Lima, menores obligaciones de revelación de información y el empleo de formatos estructurados para el trámite de inscripción mencionado. 
pensar en emplear herramientas tecnológicas hace unos años en comparación de hoy en día toda vez que los procesos cada vez son más simplificados y las posibilidades de acceso cada vez son mayores.

En ese sentido por las razones expuestas podemos considerar al crowdfunding como una alternativa de financiamiento, sin embargo a pesar de su relevancia no existe actualmente en Perú normativa aplicable que determine las disposiciones a las cuales debe sujetarse para una adecuada protección del interés público inherente en la captación de recursos ni entidad de la administración pública que cuente con facultades para autorizar aquellos mecanismos que resulten idóneos para dar a conocer su contenido.

\section{ii. Participantes del crowdfunding}

Habiendo identificado la definición del crowdfunding podemos detallar los agentes que intervienen en el mencionado financiamiento en el siguiente listado:

- Inversionistas: Identificados como las personas naturales y/o jurídicas que entregan fondos a los proyectos, ideas o actividades determinadas $\mathrm{o}$ determinables con la finalidad de obtener un beneficio que puede ser representado como una rentabilidad por su excedente de capital entregado o la preferencia en la entrega del producto o prestación del servicio elegido a través de una plataforma de crowdfunding.

- Beneficiarios: Identificados como las personas naturales y/o jurídicas que obtienen el financiamiento necesario para poder materializar sus proyectos, ideas o actividades determinadas o determinables permitiéndoles conocer con anticipación la aceptación que tiene su emprendimiento en el mercado considerando los recursos obtenidos y el número de personas que comparten su visión.

- Plataformas de crowdfunding: Son consideradas como tales las plataformas tecnológicas que permiten la conexión entre inversionistas y beneficiarios para desarrollar actividades de crowdfunding.

- Promotores: Considerados como tales a aquellas personas naturales y/o jurídicas que podrían contribuir con el diseño publicitario requerido para atraer la atención de la campaña de financiamiento y resaltarla lo suficientemente necesario para que sea identificable en las listas de proyectos disponibles en las plataformas de crowdfunding.

\section{iii. Beneficios}

Dentro de los beneficios del crowdfunding podemos identificar los siguientes:

- Para las personas naturales y/o jurídicas que cuentan con proyectos, negocios o ideas determinadas o determinables otorga el acceso a un canal que le permitiría obtener el financiamiento necesario para materializar su 
emprendimiento. Del mismo modo, al obtener recursos en una etapa previa le permite tener conocimiento de la aceptación que tiene en el mercado entre sus potenciales clientes que basándose únicamente en la información preliminar consideran atractiva y rentable la idea propuesta.

- Para los inversionistas y potenciales inversionistas les permite diversificar su portafolio de inversiones desarrollando de esa forma una eficiente gestión de riesgos.

- Para el país le otorga la posibilidad de crecimiento de la economía nacional en diversos sectores en los que se encuentren identificados los negocios, ideas o actividades determinadas y/o determinables, así como también le permite alinearse al desarrollo e implementación tecnológico que es utilizado en otros países.

\section{iv. Modalidades}

De acuerdo a lo señalado por las Naciones Unidas en su documento del 12 de junio del 2017 denominada "Financing Solutions for Sustainable Development" el crowdfunding se desarrolla en cuatro modalidades que a continuación se exponen:

- Crowdfunding de donaciones o también denominado "Donations-based": entendido como el mecanismo que tiene como finalidad donar los recursos captados sin esperar algún tipo de rentabilidad como parte del proceso. Este tipo de crowdfunding es empleado comúnmente para brindar ayuda económica en caso de desastres naturales, programas educativos, pobreza extrema, entre otros.

Por ejemplo podemos considerar como plataformas electrónicas en las que se cumplen las finalidades altruistas previamente mencionadas a: (i) JustGiving2 cuyas oficinas centrales se encuentran en Bankside, Londres y que tiene como finalidad brindar las herramientas necesarias para la recolección de fondos cuyo destino sea contribuir con donaciones a través del uso de tarjetas de crédito y débito; así como también (ii) GoFundMe ${ }^{3}$ que permite recaudar fondos cuya finaldad sea destinarlos a gastos médicos, gastos formativos, programas de voluntariado, deportes juveniles, funerales, entre otros.

- Crowdfunding de recompensas o también denominado "Rewards-based": entendido como el mecanismo que tiene como finalidad obtener una recompensa por los fondos entregados la que en la mayoría de casos es la preferencia en la entrega del producto o prestación del servicio inicialmente propuesto a través de la plataforma electrónica.

Por ejemplo podemos considerar como plataformas de este tipo a las

www.justgiving.com

www.gofundme.com 
siguientes: (i) Kickstarter ${ }^{4}$ que permite la captación de fondos para proyectos comprendidos entre distintas categorías como comics, ilustraciones, cine, arte, diseño y tecnología, juegos, música, entre otros; así como también (ii) Indiegogo ${ }^{5}$ que al igual que la plataforma previamente mencionada permite obtener recursos para financiar proyectos de distintos tipos tales como aquellos referidos a telefonía, viajes, salud y deporte, entre otros.

- Crowdfunding de crédito o también denominado "Lending-based" o "Peer-topeer": entendido como el mecanismo que tiene como finalidad otorgar préstamos a cambio del pago de los intereses respectivos.

Por ejemplo podemos considerar como plataforma de esta modalidad a KIVA ${ }^{6}$ cuya finalidad es brindar la posibilidad de acceder a préstamos brindando de esa forma oportunidades para muchas personas en todo el mundo incorporándolos en categorías de ganado, agricultura, educación, entre otros.

- Crowdfunding participativo o también denominado "Equity-based": entendido como el mecanismo que tiene como finalidad adquirir participación en el accionariado de empresas.

Por ejemplo podemos considerar como plataforma de este tipo a EquityNet ${ }^{7} \mathrm{y}$ CircleU $p^{8}$ que cuentan dentro de sus categorías la posibilidad de acceder a una sección destinada a equity.

\section{Experiencias anteriores de Crowdfunding}

La presente sección tiene como finalidad describir el tratamiento que se le otorga al crowdfunding en distintos países con el objetivo de identificar sus antecedentes para conocer sus orígenes y elementos que motivaron su creación, su evolución a fin de determinar el desarrollo que tuvo en la industria de financiamientos y las posibles iniciativas legales que pudo haber generado, así como también su situación actual con el objetivo de conocer el escenario en el que nos encontramos para una posterior comparación con su tratamiento en nuestro país.

\section{i. Países integrantes del Mercado Integrado Latinoamericano}

La integración regional bursátil latinoamericana comprende a los países México, Colombia, Chile y Perú por lo que en el marco del desarrollo de la integración objeto de comentario es conveniente identificar el tratamiento actual que se le brinda al crowdfunding a fin de identificar escenarios que podrían tomarse como referencia para implementar la regulación respectiva en nuestro país.

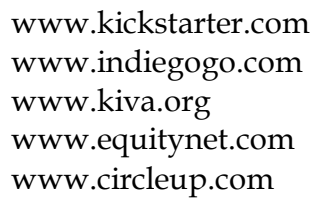


- $\quad$ En México:

En el mencionado país se publicó el 9 de marzo del 2018 la "Ley para regular las instituciones de tecnología financiera" que incorpora las disposiciones aplicables para las entidades denominadas "instituciones de tecnología financiera" como aquellas que requieren autorización previa por parte de la Comisión Nacional Bancaria y de Valores.

De igual manera, la norma previamente mencionada establece las obligaciones de revelación de información a fin de evitar que se difunda información falsa o engañosa y de esa manera difundir toda la información que sea necesaria para que los clientes de las instituciones de tecnología financiera identifiquen oportuna y adecuadamente los riesgos en operaciones de financiamiento colectivo.

- $\quad$ En Colombia:

En dicha jurisdicción se cuenta con el Decreto 1357 del 31 de julio de 2018 por el cual se modifica el Decreto 2555 de 2010 en lo relacionado con la actividad de financiación colaborativa; el cual se encuentra alineado con las actividades de promoción de la inclusión financiera y el potencial del crowdfunding en generar profundidad al sistema financiero. La mencionada norma considera que el crowdfunding permite la simplificación de procesos operativos y genera el acceso a novedosas alternativas de financiamiento.

Dentro de las disposiciones contenidas en la mencionada norma se identifica un régimen obligatorio de revelación de información, estándares operativos y de funcionamiento a ser empleados, mecanismos orientados a la protección de inversionistas y beneficiarios en el uso de las plataformas de financiamiento participativo. Cabe mencionar que la norma previamente mencionada considera inclusive disposiciones vinculadas a cautelar la prevención de lavado de activos.

La norma bajo comentario emplea el término "financiación colaborativa" con el objetivo de definir al crowdfunding señalando que implica una actividad desarrollada por entidades que cuenten con previa autorización de la Superintendencia Financiera de Colombia. Las mencionadas entidades emplean una infraestructura electrónica, páginas de internet u otro medio de comunicación electrónica que permite interconectar a aportantes y receptores en el marco de un proyecto.

La presente regulación colombiana excluye de su ámbito de aplicación al crowdfunding cuyos destinos sean donaciones y recompensas.

- $\quad$ En Chile:

En Chile no se cuenta actualmente con regulación para el crowdfunding en ninguna de sus modalidades, sin embargo un documento publicado por el Banco Central 
de Chile denominado "Desarrollo del Crowdfunding en Chile" de abril del $2018^{9}$ en el que se reconoce la importancia y el crecimiento que ha tenido durante los últimos años. La mencionada publicación reconoce que el uso del crowdfunding implica riesgos que deben ponderarse como los siguientes: riesgo de crédito, riesgo de falta de transparencia, riesgo de fraude, riesgo legal, riesgo tecnológico y riesgo de liquidez.

\section{- $\quad$ En Perú:}

Como veremos en la siguiente sección en nuestro país actualmente no se cuenta con regulación aplicable para las actividades de crowdfunding que abarca la autorización previa de entidades autorizadas a administrar las plataformas de financiamiento, obligaciones de revelación de información con la finalidad de protección de los inversionistas, entre otras.

\section{ii. Europa}

El crowdfunding se encuentra actualmente regulado y es usado como alternativa de financiamiento en diversos países de Europa tales como España, Alemania, Francia, Italia y Reino Unido. Para efectos del presente texto se abordarán tres de ellos que a continuación se mencionan:

- $\quad$ En España: El 28 de abril del 2015 se publicó la Ley N 5/2015 denominada "Ley de Fomento de la Financiación Empresarial" la que tiene como objetivo principal establecer las disposiciones aplicables para una eficiente asignación de recursos entre los agentes con capacidad de ahorro y aquellos que necesitan financiamiento. En ese orden de ideas, se incorporó en el Título V de la mencionada ley un régimen jurídico aplicable para lo que se define en dicha norma como "Plataformas de financiación participativa" o lo que conocemos comúnmente como crowdfunding. No obstante ello, la norma mencionada regula disposiciones aplicables estrictamente al crowdfunding participativo y de préstamo por su naturaleza financiera excluyendo de su ámbito de aplicación a los crowdfunding de donaciones o recompensas.

Las plataformas de financiación participativa son consideradas como aquellas que ponen en contacto a promotores de proyectos que se ven beneficiados con los recursos obtenidos e inversionistas que buscan obtener una rentabilidad con su inversión. Al respecto, el ordenamiento jurídico español identifica dos características inherentes a las plataformas de financiación como son: (i) el acceso y uso masivo de la plataforma debido a la conexión tecnológica y (ii) que no discrimina entre los inversionistas o potenciales inversionistas que podrían acceder a los proyectos, ideas y/o actividades que son previamente

9 ABARCA, Iván. Desarrollo del Crowfunding en Chile. Documentos de Trabajo del Banco Central de Chile $\mathrm{N}^{\mathrm{0}}$ 815. Información recuperada en noviembre de 2018 de: http:/ / www.bcentral.cl/documents/20143/926756/dtbc815.pdf/ef8fe943-fdf6-6352-3dcd3f9bb23f36e0 
seleccionados por su atractivo potencial.

Del mismo modo, se reserva la actividad del financiamiento participativo a aquellas entidades que son previamente autorizadas para tales fines lo que implica el previo cumplimiento de determinados requisitos orientados a cautelar la estabilidad financiera. Asimismo, la autorización genera la inscripción de la entidad autorizada en la Comisión Nacional del Mercado de Valores.

Por otro lado, se incorporan también límites de captación dependiendo del tipo de proyecto frente al que nos encontremos con la finalidad de otorgar una adecuada protección a los inversionistas participantes y el consentimiento escrito del inversionista que permita expresar que entiende los riesgos inherentes al tipo de inversión que realiza a través del financiamiento participativo o crowdfunding

Asimismo, la norma incorpora un régimen de revelación de información obligatoria por parte de las entidades autorizadas que publiciten determinados proyectos, ideas y/o actividades previamente seleccionados.

Finalmente, la citada ley confiere facultades de supervisión, inspección y sanción a la Comisión Nacional de Mercado de Valores respecto de las plataformas de financiamiento participativo y de las personas naturales y/o jurídicas que infrinjan la reserva de actividad y denominación bajo comentario, es decir, el emplear denominaciones y/o desarrollar actividades vinculadas al financiamiento participativo sin contar previamente con la debida autorización respectiva.

- En Italia: se emitió en el año 2013 la Resolución Nº 18592 mediante la cual se aprobó el "Reglamento sobre la captación de capital de riesgo mediante nuevas empresas innovadoras a través de portales en línea" mediante el cual se incorporaron las disposiciones aplicables para las plataformas de financiamiento participativo. Al respecto, la mencionada norma busca garantizar la protección de los inversionistas, incorpora normas de conducta para los gestores de dichas actividades, dispone un régimen obligatorio de revelación de información y controla el cumplimiento de las condiciones de las ofertas realizadas en dichas plataformas.

- En el Reino Unido: se cuenta también con regulación aplicable que incorpora con disposiciones orientadas a la defensa de los inversionistas, sin embargo su ámbito de aplicación comprende los crowdfunding participativo y de préstamo, excluyendo a los crowdfunding de donaciones y recompensa. Sobre el particular, cabe indicar que las disposiciones respectivas se encuentran establecidas en el documento de marzo del 2014 denominado "Policy Statement 14/4, The FCA's regulatory approach to crowdfunding over the internet, and the promotion of non-readily realizable securities by other media". 


\section{iii. En Estados Unidos}

El tratamiento otorgado al crowdfunding se identifica en la ley denominada "Jumpstart Our Bussiness Startups (JOBS) Act" promulgada en el 5 de abril de 2012 durante el gobierno del presidente Barack Obama y que tiene como finalidad promover el financiamiento de pequeñas empresas a través de la flexibilización de la regulación. En la mencionada norma se incorporó un régimen aplicable para las actividades de crowdfunding que permitía a las empresas emitir valores y que complementado posteriormente por la normativa emitida por la Securities and Exchange Comission referida al crowdfunding de participación o de equity.

Para efectos del desarrollo de las actividades de crowdfunding previamente mencionadas se requiere el registro en la Securities and Exchange Comission y la Financial Industry Regulatory Authority a las que deben presentar información referida al proyecto específico, límites referidos a sumas invertidas en el desarrollo del emprendimiento, entre otros.

\section{Implementación del crowdfunding en el Perú}

La presente sección analizará el escenario actual del crowdfunding en nuestro país considerando el marco teórico inicialmente descrito y el posterior análisis realizado de las experiencias pasadas. En ese sentido, la presente sección tiene por finalidad determinar la situación en la que nos encontramos en comparación con los países mencionados en la sección previamente desarrollada.

\section{i. Antecedentes}

De acuerdo a lo expuesto previamente en diversos países se han implementado normas cuya finalidad es brindar un escenario con reglas detalladas para una eficiente asignación de recursos en el marco de las actividades de crowdfunding o también conocido en algunos casos como financiamiento participativo. Asimismo, se ha identificado que la mayoría de regulaciones se orientan a dos tipos de crowdfunding: participativo o "Equity-based" y de préstamo o "Lending-based" por su naturaleza financiera, excluyendo a los crowdfunding de donaciones o "Donations-based" y de recompensa o "Rewards-based".

En nuestro país no contamos actualmente con una normativa aplicable al crowdfunding, sin embargo se encuentra vigente el Decreto Legislativo $N^{\circ}$ 861, Ley del Mercado de Valores la que abarca la supervisión a las ofertas públicas de valores mobiliarios y cuyo cumplimiento se encuentra a cargo de la Superintendencia del Mercado de Valores.

En ese orden de ideas, la mencionada Ley del Mercado de Valores podría resultar ser la norma adecuada para incorporar disposiciones aplicables al crowdfunding a través de una modificación normativa toda vez que de esa forma en un mismo cuerpo normativo los agentes que deseen obtener financiamiento pueden considerar dicha alternativa como una opción adicional. 
Asimismo, considerando que el cumplimiento de las disposiciones contenidas en la Ley del Mercado de Valores se encuentra a cargo de la Superintendencia del Mercado de Valores resulta ser ella misma también la entidad de la administración pública adecuada para también verificar el cumplimiento de las disposiciones aplicables a las actividades de crowdfunding.

Por otro lado, otro camino sería la aprobación de una ley nueva específicamente destinada a detallar las disposiciones aplicables al financiamiento participativo y delegar en la Superintendencia del Mercado de Valores la facultad de regular vía reglamento los aspectos complementarios que resulten necesarios para cumplir con el objetivo del legislador.

Sin perjuicio de lo señalado, resulta importante mencionar que las modificaciones normativas previamente señaladas deben contar con un adecuado análisis de impacto regulatorio con la finalidad de evitar la sobreregulación. Al respecto, debe considerarse que la sobreregulación resulta un desincentivo para la promoción e inclusión financiera en las actividades de crowdfunding no solo para los potenciales inversionistas sino también para aquellas personas naturales y/o jurídicas que podrían dejar de considerar el financiamiento participativo como una alternativa atractiva.

En ese sentido, el reto en nuestro país durante los últimos años ha sido determinar si existe o no necesidad de aprobar una norma para el crowdfunding y la vía adecuada para hacerlo.

\section{Escenario actual}

Actualmente conforme ha sido mencionado en nuestro país no existe normativa aplicable al crowdfunding, sin embargo el 27 de junio del 2018 fue presentado al Congreso de la República el Proyecto de Ley 3083/2017-CR denominado "Proyecto de Ley mediante el cual se propone la Ley que regula el régimen jurídico de las plataformas de financiamiento participativo - PFP"10.

El Proyecto de Ley bajo mención propone definir al crowdfunding como "financiamiento participativo" señalando que conlleva el empleo de plataformas de páginas web u otros médicos electrónicos por personas naturales y/o jurídicas que califiquen como inversionistas y a personas que propone denominar "promotores" que necesiten financiamiento para el desarrollo de sus proyectos.

El texto del Proyecto de Ley emplea términos utilizados también en la regulación extranjera mencionada en el presente documento por lo que la intención es alinearlo con la terminología empleado en el ámbito internacional.

Respecto de los aspectos relevantes del Proyecto de Ley cabe señalar los que a

10 El Proyecto de Ley bajo mención fue propuesto en ejercicio del derecho que confieren los artículos 102, inciso 1) y 107 de la Constitución Política del Perú, así como también el numeral 2 del artículo 76 del Reglamento del Congreso de la República. 
continuación se expone:

- Propone extender su ámbito de aplicación únicamente para dos tipos de crowdfunding o como lo denomina "financiamiento participativo" tales como: (i) crowdfunding de inversión o "Equity-based" y (ii) crowdfunding de préstamo o "Lending-based".

En ese sentido, excluye expresamente de su ámbito de aplicación a la búsqueda de financiamiento de: (i) crowdfunding de donaciones o "Donationsbased", (ii) venta de bienes y servicios, (iii) préstamos o mutuos sin intereses y (iv) Otras modalidades no comprendidas expresamente en la mencionada propuesta normativa.

- Propone la creación de la figura jurídica de "Plataformas de financiamiento participativo" estableciendo los requisitos que debe presentar para que se le otorgue la respectiva autorización de funcionamiento.

$\mathrm{Al}$ respecto, uno de los requisitos propuestos más importante es contar con una póliza de seguros cuyo monto varía en virtud del monto del proyecto. En ese sentido, el rango de los montos de las pólizas se da en tres (3) topes: 60 UIT, 90 UIT y 150 UIT.

- Propone detallar las características que deben considerarse para clasificar a determinadas actividades como financiamiento participativo e inclusive si calificarían como financiamiento participativo a través de préstamos o valores.

Sobre el particular, ello contribuiría en gran medida en su aplicación inicial con la finalidad de delimitar determinadas actividades dentro de la categoría idónea.

- Propone señalar expresamente que el financiamiento participativo a través de valores que se realice en cumplimiento de las disposiciones contenidas en la normativa propuesta no tenga la calificación de oferta pública bajo las normas de la Ley del Mercado de Valores y sus respectivos reglamentos.

Cabe mencionar que ello contribuiría a delimitar la aplicación de la normativa respectiva en caso nos encontremos frente a situaciones similares cuya única diferencia sea el medio empleado para obtener el financiamiento respectivo.

- Proponer señalar que los inversionistas no tendrán la calidad de consumidores frente a los promotores, sin embargo quienes sí tendrían dicha condición serían los inversionistas y promotores con las plataformas de financiamiento participativo.

Ello contribuiría a extender las disposiciones contenidas en el Código de Defensa y Protección del Consumidor a las relaciones generadas en el marco de las actividades de financiamiento participativo en el sentido expuesto en el párrafo precedente. 
- Propone establecer un régimen de revelación de información con la finalidad de cautelar los intereses de los promotores e inversionistas.

De esa forma se contribuye con la cautela del interés público que justifique la intervención del Estado mediante el otorgamiento de una autorización de funcionamiento a las plataformas de financiamiento participativo.

- Propone otorgarle a la Superintendencia del Mercado de Valores las facultades de supervisión y sanción respecto de las actividades desempeñadas por las plataformas de financiamiento participativo. Asimismo, incorpora un listado de infracciones y sanciones a ser implementadas por la SMV.

En ese sentido, se delega en una entidad de la administración pública la verificación del cumplimiento de las disposiciones aplicables para lo que sería una nueva alternativa de financiamiento.

Por otro lado, resulta importante señalar que la Superintendencia del Mercado de Valores mediante Oficio 070-2017-SMV/02 del 6 de marzo de 2017 remitió al Ministerio de Economía y Finanzas un proyecto de ley que buscaba establecer el marco regulatorio y de supervisión que permita la colocación, adquisición u oferta de valores mobiliarios a través de las plataformas de financiamiento participativo de valores mediante la incorporación del Título XVII - "Plataformas de Financiamiento Participativo de Valores" a la Ley del Mercado de Valores, Decreto Legislativo 861.11

De igual manera, la Superintendencia de Banca, Seguros y Administradoras de Fondos de Pensiones el 18 de octubre de 2017 mediante presentación a la Comisión de Economía, Banca, Finanzas e Inteligencia Financiera del Congreso de la República ${ }^{12}$ señaló que respecto del financiamiento participativo o crowdfunding encontraban potencial que generaba los siguientes beneficios: (i) puede aumentar rendimientos para el inversionista y (ii) puede disminuir costos para los demandantes de crédito así como facilitar acceso al crédito. No obstante ello, en la referida presentación se precisó también que genera riesgos que requieren ser regulados tales como: (i) pérdida del capital invertido por riesgo de crédito, operacional y crimen cibernético, (ii) la información a los inversionistas puede no ser transparente y alertar de los riesgos y (iii) podría usarse capital ilícito para financiar actividades reales o no existentes.

Posteriormente, la Superintendencia del Mercado de Valores, la Superintendencia de Banca, Seguros y Administradoras de Fondos de Pensiones y el Banco Central de Reserva del Perú producto de labores de

11 Memoria Anual de la SMV - 2017 http://www.smv.gob.pe/ConsultasP8/temp/2018019251-Memoria_SMV_2017.pdf

12 http://www.congreso.gob.pe/Docs/comisiones2017/Comision_de_Economia/files/ exposiciones/pres_sbs.pdf 
coordinación, consulta e intercambio de información elaboraron un proyecto de ley que busca regular las plataformas de financiamiento y que fue presentado al Ministerio de Economía y Finanzas en octubre del 2018. Cabe indicar que de acuerdo a la información de la presentación publicada en el portal web de la Superintendencia de Banca, Seguros y Administradoras de Fondos de Pensiones ${ }^{13}$ dentro del marco del mencionado proyecto de ley se encontrarían los aspectos que a continuación se detallan:

- Regular las plataformas de financiamiento participativo o denominado crowdfunding abarcando en el sector financiero las modalidades de: (i) préstamos e (ii) inversiones en capital y/o deuda. Al respecto, la definición que se otorgaría al financiamiento participativo sería la siguiente: "Pequeñas cantidades de dinero provenientes de un gran número de personas para financiar un proyecto, negocio u otra necesidad, a cambio de cierta rentabilidad".

De igual manera, se incluiría también a las modalidades del crowdfunding del sector no financiero como son: (i) donaciones y (ii) recompensas. Sobre el particular, conforme ha sido desarrollado de manera previa ambas modalidad son reconocidas a nivel mundial inclusive por las Naciones Unidas en su documento del 12 de junio del 2017 denominada "Financing Solutions for Sustainable Development".

Por otro lado, el proyecto de ley también incluiría regulación aplicable a las plataformas de financiamiento no participativo tales como: (i) préstamos entre un solo ofertante y un solo demandante y (ii) créditos otorgados como fondos propios de la empresa que gestiona la plataforma.

- Otorgar a la Superintendencia del Mercado de Valores la facultad de regulación y supervisión de las plataformas de financiamiento participativo. Sobre el particular, al otorgar dichas facultades a la SMV se buscaría otorgar seguridad jurídica al público en general toda vez que uno de los objetivos de la mencionada entidad pública es la protección a los inversionistas.

- Encontrar un balance entre los objetivos de protección y control de riesgos, y la flexibilidad necesaria para que la innovación financiera pueda desarrollarse.

Cabe mencionar que a la fecha de redacción del presente documento el proyecto de ley aún se encuentra en evaluación por parte del Ministerio de Economía y Finanzas que será quien decidirá eventualmente su contenido y posterior presentación ante el Congreso de la República del Perú.

13 Fintech y consideraciones para su regulación. Mayo, 2018. http://www.sbs.gob.pe/Portals/0/jer/SUPERVISION_OP1/LimaFintechForum_SBS_Mayo_2 018.pdf 


\section{Visión a futuro}

Luego de haber desarrollado el marco conceptual del crowdfunding, analizado las experiencias de países sobre el tratamiento que le otorgan al financiamiento participativo y mencionado algunos de los aspectos más relevantes que son propuestos en el Proyecto de Ley 3083/2017-CR denominado "Proyecto de Ley mediante el cual se propone la Ley que regula el régimen jurídico de las plataformas de financiamiento participativo - PFP", así como también el marco del proyecto de ley elaborado por la Superintendencia del Mercado de Valores, la Superintendencia de Banca, Seguros y Administradoras de Fondos de Pensiones y el Banco Central de Reserva del Perú; resulta importante señalar que el reto de nuestro país es asegurarse que las modalidades de crowdfunding a regular no sean confundidas con las formas de captación de recursos actualmente vigentes y que son realizadas por entidades reguladas tanto por el lado del sistema bancario como también del mercado de valores.

Otro aspecto importante a considerar son las obligaciones de revelación de información que contribuyen a la adopción de decisiones de inversión y disminuye el riesgo de falta de transparencia.

De igual manera, resulta importante señalar que otorgar responsabilidades a las entidades autorizadas que administren las plataformas de financiamiento permitirá cautelar un adecuado funcionamiento de dichas actividades y de esa forma evitar desarrollar actividades de fraude en la captación de recursos del público.

Finalmente, el crowdfunding tiene un largo camino por recorrer aún en nuestro país, sin embargo si consideramos que la tendencia actual en el mundo es regular dichas actividades debe realizarse un análisis de impacto regulatorio que justifique en nuestro ordenamiento jurídico su implementación y que en consecuencia profundice nuestro sistema financiero. 